\title{
Face facts: Even nonhuman animals discriminate human faces
}

\author{
Edward A. Wasserman ${ }^{1}$ \\ Published online: 15 July 2016 \\ (C) Psychonomic Society, Inc. 2016
}

Summary Humans are commonly believed to have evolved specially adapted neural systems for processing the rich and complex content of faces. However, nonhuman animals - including fish - have also shown a well-developed capacity for discriminating human faces, raising important questions concerning the uniqueness and mechanisms of human face perception.

Keywords Face perception - Comparative cognition . Evolution $\cdot$ Neural systems

Face perception plays a vital role in our everyday social functioning. An interesting aspect of face processing is that an individual human face can be simultaneously classified in multiple ways: identity, gender, age, and emotional expression. The richness and capacity of human face perception is considered to be one of the most impressive achievements of our visual system.

Growing behavioral and neurobiological evidence has been interpreted as supporting the existence of a specialized face processing module in the human brain - the fusiform face area. As with other purportedly specialized systems, it has been suggested that the face processing system has been adapted for this particular task because of strong evolutionary pressures and, further, that some of its neural mechanisms are innate, inheritable, and uniquely human.

One strategy for determining whether a particular perceptual or cognitive capacity results from a specialized adaptation or from one or more general systems is to compare the behavior of distantly related species that share a remote common ancestor. Such distantly related species are likely to share only general mechanisms of behavioral adaptation that proved functional in that common ancestor. These general mechanisms ought to be useful in solving problems that are important for survival across a broad spectrum of environments rather than to be deployed for solving a highly specific problem like face recognition. Such general perceptual and cognitive mechanisms ought to be con-

Edward A. Wasserman

ed-wasserman@uiowa.edu

1 The University of Iowa, Iowa City, IA, USA served across evolution. Adaptive specializations, on the other hand, ought to be evident in only one of a pair of distantly related species, unless similar evolutionary pressures encouraged convergent evolution of a similar system (Papini, 2002).

Newport, Wallis, Reshitnyk, and Siebeck (2016) adopted the tactic of studying a distant evolutionary relative of humans - the archerfish - to see whether members of this species can discriminate human faces. Using a simple two-alternative forced-choice task, Newport et al. found that archerfish can discriminate photographs from as many as 44 different human faces, even when controlling for head shape, image color, and brightness cues. These results indicate that a vertebrate lacking a neocortex and with no evolutionary or individual history of discriminating human faces can nevertheless learn to do so.

These results should not be construed to mean either that archerfish process human faces in the same way as do people or that people lack specialized processes for face perception. Considerable current research in comparative psychology is based on the idea that any form of complex perception or cognition may arise from a number of subprocesses, with some being limited to a particular species and others being more general. From this perspective, it is entirely possible that both specialized and general processes participate in human face recognition; it remains a key empirical challenge to determine the respective contributions of each kind of process to this complex feat.

The archerfish study further emphasizes the importance of avoiding two interpretive errors when considering the evolution of a specialized human face processing system. First, evidence for a specialized process does not require that such a process is somehow free from the influence of more general processes, with only human faces engaging this specialized system. Second, the evolution of a face recognition system may not have involved the specialization of perceptual processes alone. The system may also have involved the specialization of facial muscles to transmit signals that could be readily decoded by already functioning visual processes.

A popular practice among researchers of perception and cognition is to hypothesize many different "specialized mechanisms" to explain their experimental data. These speculations are sometimes insufficiently supported by empirical data. 
Comparative research can help considerably in advancing from mere speculation about the evolutionary roots of human cognition and perception to a real appreciation of these processes.

Comparative studies involving closely related species, such as nonhuman primates, certainly have the potential to enrich our understanding of the origin and mechanisms of such specialized systems. However, primate studies leave open the question as to whether the system under study is limited to species that are closely related to humans. In order to eliminate the possibility that more general, evolutionarily conserved processes underlie a particular behavior, the study of distantly related species is required. Only when there is evidence that such general principles cannot explain the behavior of interest is it prudent to conclude that dedicated mechanisms have arisen as the result of specific evolutionary pressures.

Just how we should approach those comparative studies merits further discussion. Here, the views of two historical figures are pertinent and informative.

Michele de Montaigne (1533-1592) was a Renaissance philosopher. In his Apologie de Raimond Sebond (1580), Montaigne argued that of all earth's many creatures, we humans are the most miserable and frail, yet also the most arrogant. That arrogance leads us to ascribe divine attributes to ourselves and to separate ourselves from all other creatures.

Montaigne raised penetrating questions about this arrogant placement of humans apart from and above all other animals. Is it really so easy to say with certainty what sets us apart from animals? By what comparisons do we ascribe brutishness only to them? Montaigne believed that skeptical inquiry into animal behavior can provide answers to these two profound questions.

From reading ancient texts, Montaigne reasoned that animals communicate socially, exhibit various forms of craftsmanship, and display signs of logical decision making. He then proposed a rule: From like results we must infer like faculties. Thus, humans and animals must obey the same laws of nature, prompting the humbling conclusion that there is no special place for humans among all of nature's creatures.

Montaigne's natural philosophy is certainly appealing in today's zeitgeist, but how can we discriminate profound from superficial resemblance? After all, seeing is notalways believing. Large, silvery predators swim in the sea, but sharks are fish and dolphins are mammals. Bats, budgerigars, and bees all fly, but these animals are mammals, avians, and insects, respectively.

In fact, there is no sure and simple way to discriminate profound from superficial resemblance. Yet a hint from America's first great psychologist suggests one way we might be able to do so.

William James (1842-1910) complained that "it is the bane of psychology to suppose that where the results are similar, processes must be the same" $(1890$, p. 528). He clearly took exception to Montaigne's earlier adage.

James underscored this interpretive problem by arguing that "psychologists are too apt to reason as geometers would, if the latter were to say that the diameter of a circle is the same thing as its semi-circumference, because, forsooth, they terminate in the same two points" (1890, p. 528). This geometrical argument suggests a promising, practical solution to the problem of deciding whether the same process underlies similar behaviors in different organisms.

Suppose that we systematically vary some independent variable across many - not just two - different parametric values and observe the effects of those variations on the behavior of different species of animals. Now, suppose that those parametric functions closely parallel one another. Most researchers would agree that such striking "parametric parallels" would be extremely unlikely to have arisen by chance; instead, those parallels suggest a common process at work in the different species. Thus, parametric study is not just workmanlike psychological science - it is vital to sound comparative behavioral study. The lovely research on short-term memory in pigeons, monkeys, and people by Wright, Santiago, Sands, Kendrick, and Cook (1985) testifies to the power of this approach.

Other, more strategic techniques can be deployed to explore parallels between human and animal behavior. Germane to the topic of this Outlook piece, Gibson, Wasserman, Gosselin, and Schyns (2005) trained pigeons and people to discriminate photographs of human faces that displayed (a) a happy or a neutral expression or (b) a man or a woman. After training, the authors used a procedure called Bubbles to pinpoint the features of the faces that both species used to make these discriminations. Bubbles analysis revealed that the features used to discriminate happy from neutral faces were different from those used to discriminate male from female faces. Furthermore, the features that pigeons used to make each of these discriminations overlapped those that humans used. These results document that new and powerful analysis techniques can be effectively applied to comparing the perceptual and cognitive process of humans and nonhuman animals.

Expect future research to more fully explore and elucidate the possible parallels between human and animal behavior. Also expect innovative methods to elucidate the neural and behavioral mechanisms of perception and cognition.

\section{References}

Gibson, B. M., Wasserman, E. A., Gosselin, F., \& Schyns, P. G. (2005). Applying bubbles to localize features that control pigeons' visual discrimination behavior. Journal of Experimental Psychology: Animal Behavior Processes, 31, 376-382.

James, W. (1890). The principles of psychology (Vol. 1). London, UK: Henry Holt.

Newport, C., Wallis, G., Reshitnyk, Y., \& Siebeck, U. E. (2016). Discrimination of human faces by archerfish (Toxotes chatareus). Scientific Reports. doi:10.1038/srep27523

Papini, M. R. (2002). Pattern and process in the evolution of learning. Psychological Review, 109, 186-201.

Wright, A. A., Santiago, H. C., Sands, S. F., Kendrick, D. F., \& Cook, R. G. (1985). Memory processing of serial lists by pigeons, monkeys, and people. Science, 229, 287-289. 